\section{Electron Microscopy Vacuum Pump Basics}

\author{
Howard Tring \\ Vacuum and Low pressure Consulting, Spring City, PA \\ tringenterprises@hotmail.com
}

\section{Q: What do an ATM, a TEM and a CAT SCAN have in com- mon?}

\section{A: They are all sophisticated instruments with machinery behind the wall.}

The fact that the machinery is "behind the wall" or out of sight may mean that it is not being looked after as well as it should. Do you have a regular schedule to check the vacuum pumps and accessories in your microscopy lab, or do you wait till it fails? Do you keep a service record of the repairs done to your vacuum pumps and the vacuum levels measured after each service? In a department where there are instruments that last ten years or more and the operators and technicians regularly change it makes good sense to keep a record of the vacuum pumps oil changes, service and performance.

In my experience with many industrial plants, preventive maintenance plans are an ideal situation when times are good. As soon as business slows, or competition causes "lean and mean" operation the maintenance schedule is gone. When it breaks we fix it, becomes the mode of operation. In many research departments or laboratories, there is often no one "in charge' of vacuum pumps. Maintenance and repairs are carried out by the most mechanically inclined technician, perhaps jobbed out to the Chemistry Department workshop (they have plenty of vacuum pump repair experience), sent back to the manufacturer or shipped to a local vacuum pump service facility.

In a microscopy lab the vacuum pumps are expected to run $24 / 7$, in many cases for six months to a year before the system may be shut down for some other work to be done on it. The pumps used are designed for constant running but do need some attention paid to them. If a vacuum pump fails it may cause other costly repairs to be needed to the microscope.

Some of the information to follow may be "old hat" to those readers with experience using electron microscopes or other surface science instruments. I hope that it will provide some basic vacuum system information for "newbies" and serve as a reminder to the "oldies" in the department.

\section{What vacuum pumps are used on electron micro- scopes?}

In my early days in the vacuum pump industry mercury or oil diffusion pumps and v-belt drive style oil sealed rotary vane (OSRV) vacuum pumps were used. Most readers will be aware of the hazards of mercury, but a large European manufacturer used a small mercury diffusion pump into the 1970s. When silicon oil became the standard fluid for oil diffusion pumps it was possible to operate an electron microscope vacuum system with very little backstreaming of oil molecules when the system was operated correctly.

It wasn't until the 1990s that turbomolecular vacuum pump technology advanced to the point where "turbo pumps" were reliable. Bearing design was the weak point of these vacuum pumps and short bearing life made them an expensive proposition for many years. The turbomolecular vacuum pump still requires a two-stage oil sealed rotary vane vacuum pump attached to it, but by the 1990s these were mostly of the direct drive design.

During the last twenty years, vacuum pump technology has continued to advance. Many of the advances have been driven by the harsh vacuum processes in the semiconductor industry. The fabrication areas (Fabs) that you may see pictures of all seem nice and clean. However, some of the processes on silicon wafers create a lot of chemical vapors and tiny particulates that all move down the vacuum pipelines and through the vacuum pumps. Dry vacuum pumps have been designed specifically to handle these dirty applications. Some newer turbomolecular vacuum pumps have a higher compression ratio and have an exhaust pressure of around one torr. This allows an oil free mechanical vacuum pump to be used for the final gas compression to atmospheric pressure. One advantage here is that this backing pump is a "dry vacuum pump" with no oil in the pumping volume.

I've introduced some terms here that some readers may not be familiar with. The next few paragraphs will try to explain how it all comes together.

\section{What do the vacuum pumps do?}

Vacuum pumps remove gas molecules from a vacuum chamber and reduce the pressure to a lower level than the surrounding atmospheric pressure. There are many types of vacuum pump; small and large; single pumps and combinations; capable of reaching rough, medium, high or ultra high vacuum ranges. Although we call them "vacuum pumps", most of them actually compress the gas molecules from a low pressure up to a higher pressure. The compression ratio of a vacuum pump can be calculated by taking the exhaust pressure and dividing it by the pump's lowest inlet pressure.

Ranges of vacuum are generally shown as follows:

- Rough Vacuum or Low Vacuum: From atmospheric pressure to about 1 torr.

- Medium Vacuum: From about 1 torr to about $1 \times 10^{-3}$ torr (0.001 torr).

- High Vacuum: From about $1 \times 10^{-3}$ torr to about $1 \times 10^{-7}$ torr.

- Ultra High Vacuum: Pressures lower than $1 \times 10^{-7}$ torr.

Remember that LOW pressure $=\mathrm{HIGH}$ vacuum and, $\mathrm{HIGH}$ pressure $=\mathrm{LOW}$ vacuum .

\section{Cross-section through a typical OSRV vacuum pump}

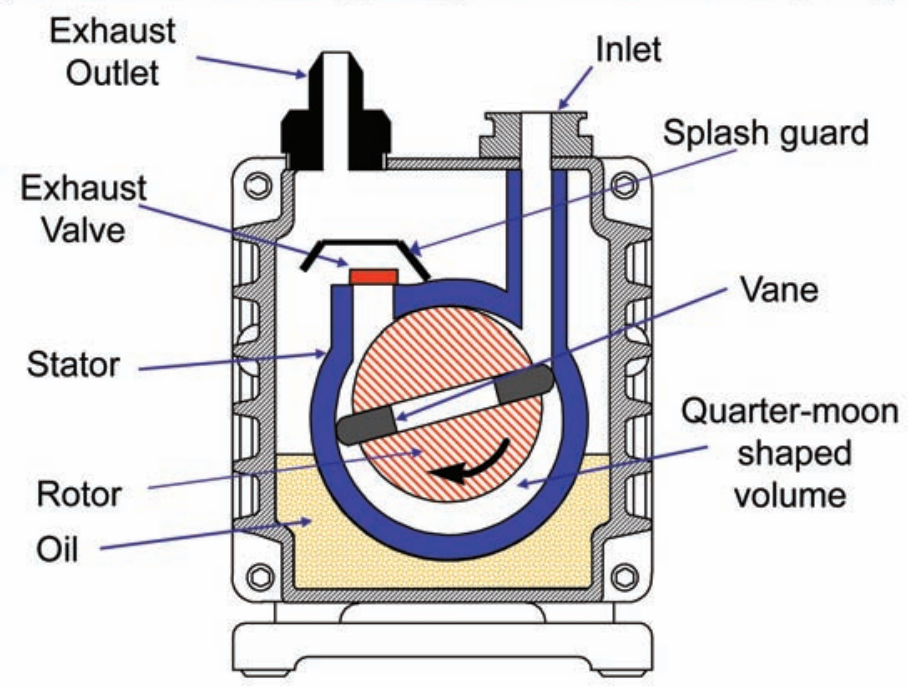




\section{Let 4pi take you beyond the capability of any SEM/STEM on the market with}

RevolutionEDX

X-ray Microanalysis Systems optimized for productivity \& ease of use

- Silicon-drift technology detectors

Liquid Nitrogen-free

High-throughput

10 or $30 \mathrm{~mm}^{2}$ sensor

- Work over a network via Gigabit Ethernet

- Easy to use: one-click acquisition for spectra, images, maps, spot probes and line scans

- Dynamic Dwell Modulation ${ }^{\mathrm{TM}}$ - spend maximum time on areas of interest while continuing to obtain $x$-ray data from the entire area

- Robust auto peak-ID based on real-time full deconvolution of acquired x-ray spectra
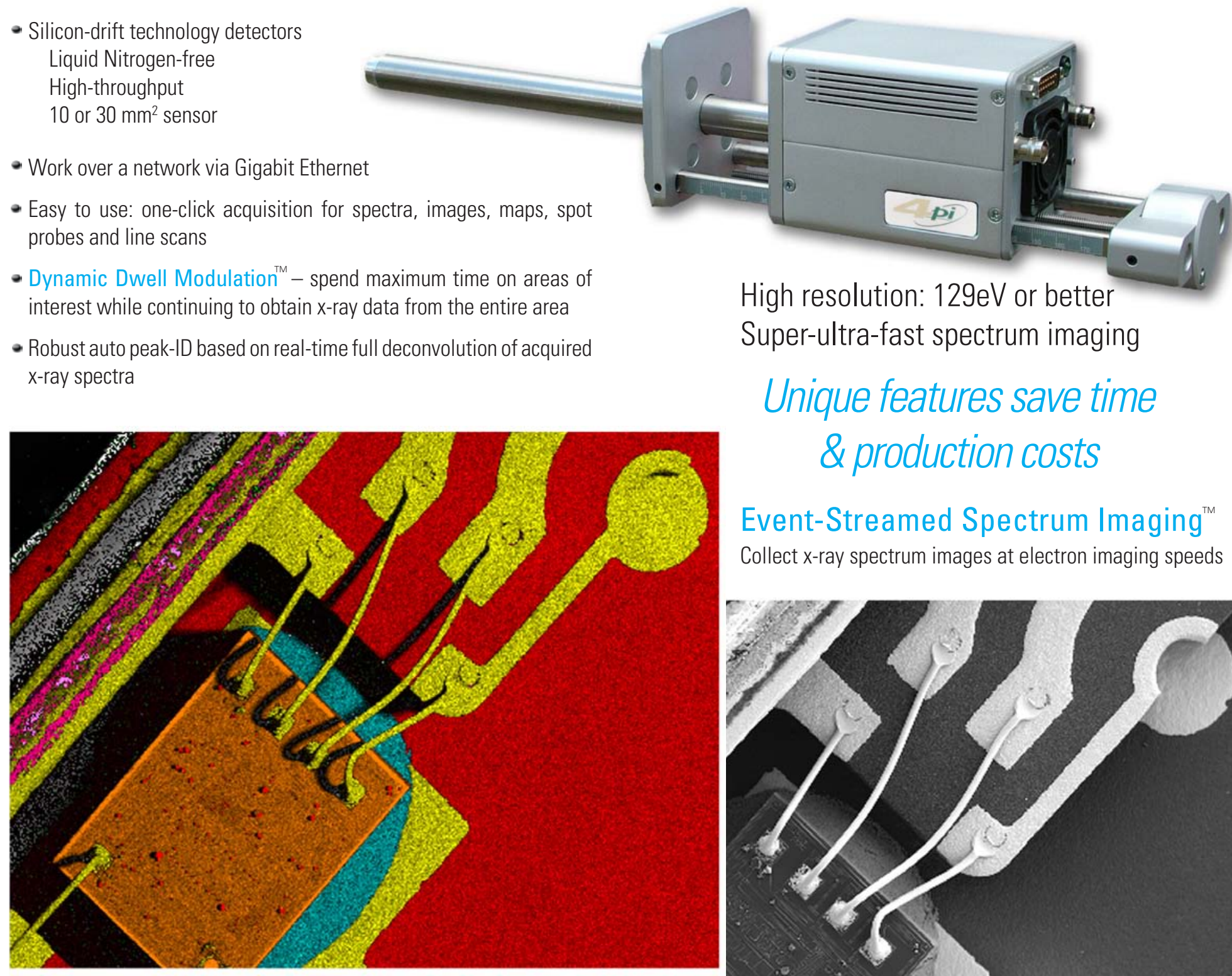

High resolution: $129 \mathrm{eV}$ or better

Super-ultra-fast spectrum imaging

Unique features save time

\section{$\&$ production costs}

\section{Event-Streamed Spectrum Imaging}

Collect $\mathrm{x}$-ray spectrum images at electron imaging speeds

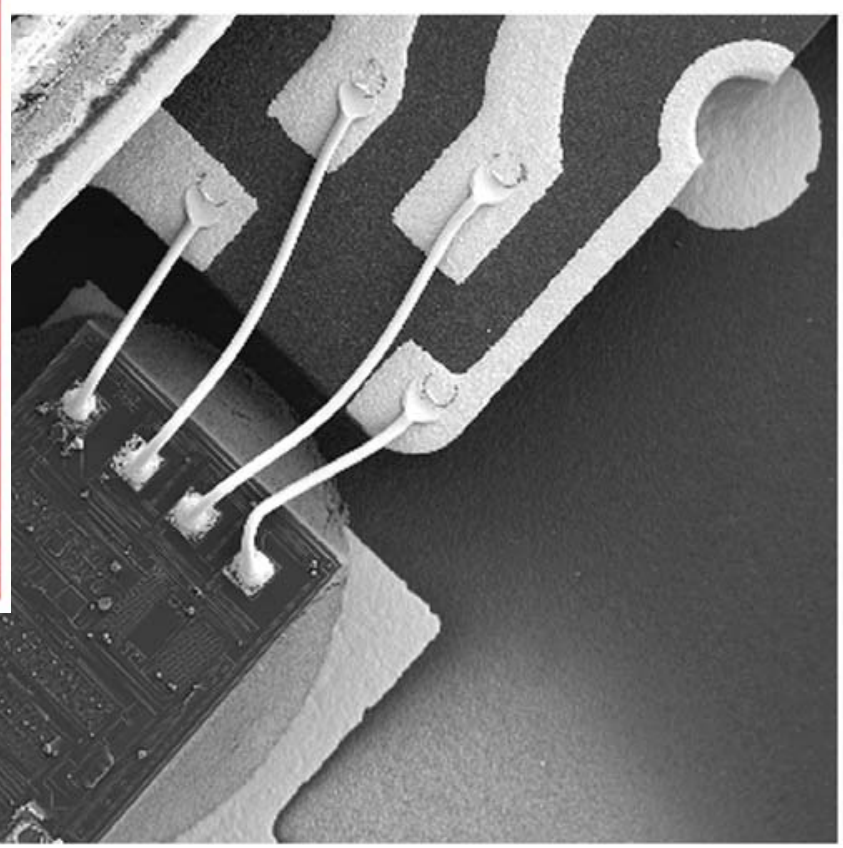

Available TODAY only from

Spatial Frame Lock

Real-time electron-beam drift correction
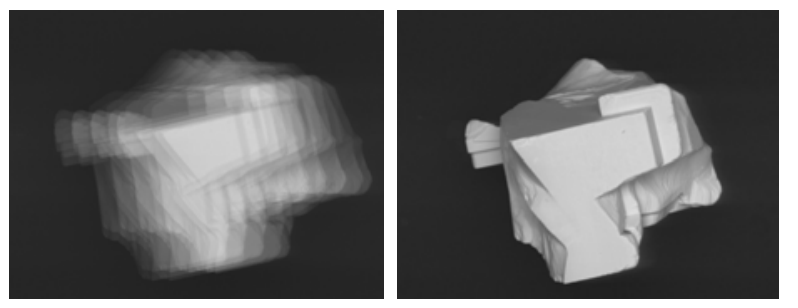

www.4pi.com・sales@4pi.com

4pi Analysis, Inc. 919.489.1757

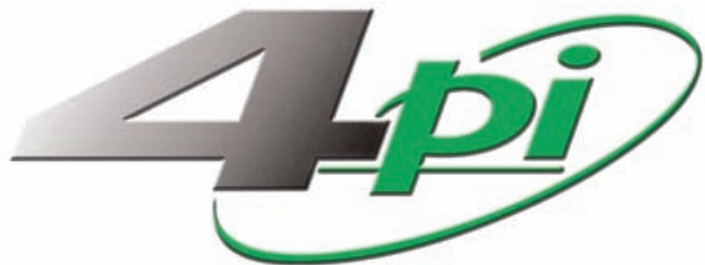


Also: Most vacuum measurements in scientific applications use absolute vacuum units. That means that the vacuum gauges read from standard atmospheric pressure down to absolute zero pressure (not attainable).

- Standard Atmospheric Pressure $=14.7$ pounds per square inch absolute. (psia)

- Std Atmospheric Pressure $=760$ torr (US) $=1,013 \mathrm{mbar}(\mathrm{EU})$ $=101,325$ pascals (ISO).

Most electron microscopes require two vacuum pumps operating in series to create the low pressure necessary for the instrument to operate. The pressure level has to be low enough that the electrons will pass across the system without colliding with a molecule of gas. It is the same technology, reducing the density of the gas, which allows the old cathode ray tubes used in televisions and computer monitors to work.

The first part of the evacuation cycle is carried out by the rotary vane pump. It can reduce the pressure from atmospheric pressure down to about $5 \times 10^{-3}$ torr. At that pressure, most of the air molecules have been removed and have been exhausted back into the atmosphere at the exhaust outlet of the vacuum pump. (The exhaust will usually have an exhaust mist filter added as an accessory to capture any fine oil mist that is entrained in the flow of air molecules.) Once the pressure is reduced below the vapor pressure of water at the temperature inside the system (about 18 torr at room temperature) molecules of water vapor on interior surfaces of the system will start to release and be pumped away. Several factors limit the inlet pressure of the oil sealed rotary vane pump to about $1 \times 10^{-3}$ torr.

To reduce the pressure to high or ultra high vacuum ranges a secondary vacuum pump is needed, this is generally a turbomolecular vacuum pump. This vacuum pump has a relatively small electric motor built into the body of the pump to turn a multi-bladed rotor at very high speed. It is not switched on until the primary (rotary vane) pump has reduced the pressure to less than 1 torr. Above one torr there are many more gas molecules still present in the system and "pumping" them can overload the small motor. The turbomolecular vacuum pump can remove enough of the remaining gas molecules that the pressure could approach $1 \mathrm{x}$ $10^{-10}$ torr. The lowest pressure attainable in a system depends on the size, design and materials of the chamber; and assumes that there are no leaks or contaminants in the system.

\section{How do the vacuum pumps work?}

Very simply, the oil sealed rotary vane pump has sliding vanes in a slotted rotor, set eccentrically inside a stator. The resulting open volume inside the pump is shaped like a quarter-moon. The rotor turns and the vanes slide in and out of the rotor slots so that their outside edge always touches the inside surface of the stator. The vanes form two volumes, sealed by oil films, which increase and decrease in size during each revolution. Gas molecules from the system are expanded into this volume at the pump inlet, trapped between the vanes and then compressed up to atmospheric pressure and exhausted. This is explained nicely by Boyle's Law, the pressure and volume of a fixed mass of gas vary in direct proportion $\left(\mathrm{P}_{1} \mathrm{~V}_{1}=\mathrm{P}_{2} \mathrm{~V}_{2}\right)$. Each rotation of the rotor reduces the system pressure by a very small amount. As these pumps rotate at about $1750 \mathrm{rpm}$, the pressure inside the electron microscope is reduced to the low pressure needed to move to the next stage of evacuation within a few minutes.

\section{A turbomolecular pump rotor}

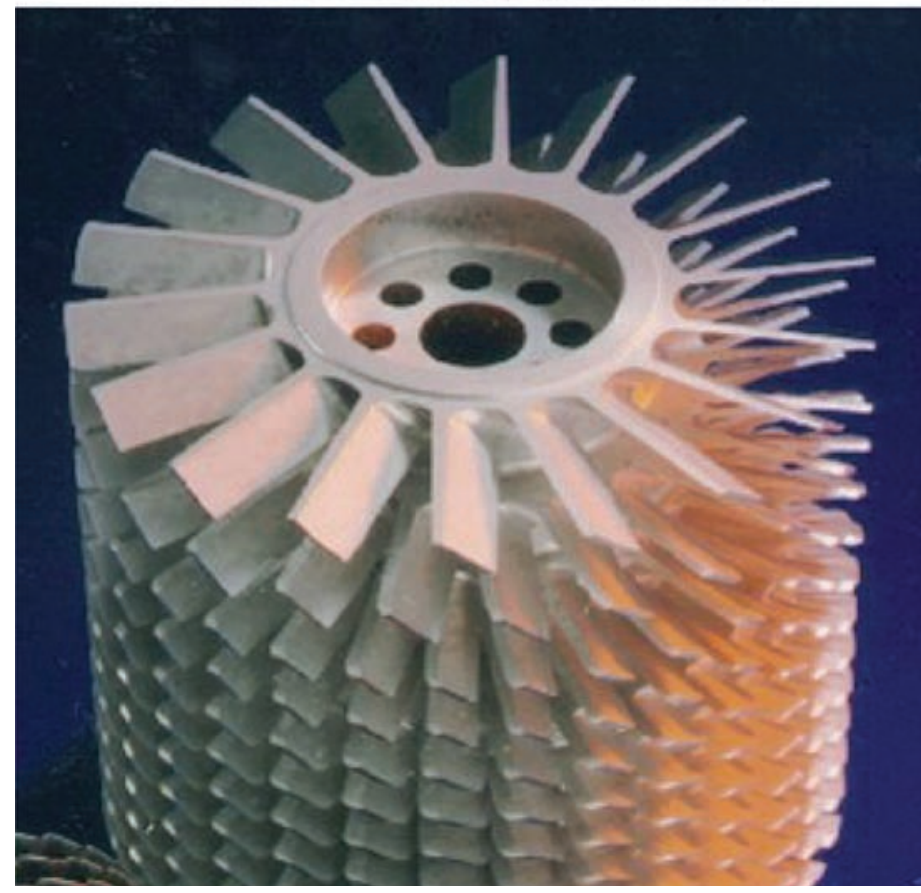

The turbomolecular pump works very differently. The multi bladed rotor in this pump has no lubrication in the pumping volume. The bearings may have oil or grease lubrication but this is outside the vacuum area. It is called a "dry pump", which means it has no lubrication in the vacuum volume. The rotor may rotate at up to $90,000 \mathrm{rpm}$ for a small pump (70 $\mathrm{mm}$ inlet diameter) and this speed allows the blades to redirect the movement of the gas molecules entering the pump into a downward (into the pump) direction. The gas molecules will be compressed through a series of angled blades and will reach the exhaust area. The rotary vane pump is connected by a vacuum line to the turbomolecular pump exhaust connection. The gas will pass through the rotary vane pump, be compressed up to atmospheric pressure and exhausted back into the atmosphere.

The turbomolecular vacuum pump is usually mounted as close to the vacuum area of the electron microscope as possible. The oil sealed rotary vane vacuum pump, sometimes referred to as the mechanical pump, is often sitting on the floor underneath or behind the instrument. In the case of a high magnification electron microscope, where vibration may affect the image, the vacuum pump may be situated away from the instrument with the vacuum line passing through an anti-vibration device. In this application the turbomolecular vacuum pump will also be a magnetic bearing design with very low vibration characteristics.

\section{Why do the vacuum pumps need some TLC?}

Oil sealed rotary vane vacuum pumps need service to look after the following main areas of concern.

- The high vacuum oil becomes contaminated, slowly oxidizes due to the heat generated at the blade tips, and loses its lubricity.

- The shaft seals harden with time and exposure to hot oil and may leak.

- Exhaust valves open and close frequently and may work harden and leak.

- Oil mist filter elements require replacement. 
Turbomolecular vacuum pumps have the following limitations:

- The high speed bearings have an effective life of about 20,000 hours.

- Contamination, although unlikely in a clean application, may cause the rotor to become unbalanced. This will lead to bearing failure and possibly worse.

- Any solid item falling into the pump inlet will cause major damage. (An inlet screen is always recommended.)

- Bearing changes are best carried out in a clean workshop and special tools, i.e. at the factory. Some rotors require rebalancing after a bearing change and this requires special equipment. Changing bearings on these costly products yourself is a risk.

- If the turbomolecular pump is a magnetic bearing version, the electronic controller is matched or tuned to the pump. Both items should be returned to the factory for service.

\section{Who should look after the vacuum pumps?}

There are four main options when you have mechanical vacuum pumps that require service.

- Fix it yourself, if you have the spares kits, fresh high vacuum oil, the workshop space, the mechanical knowledge and can spare the time away from your specialty.

- Send it to the Research Laboratory or University Science workshop where the technicians there will service it for you. That may take a few weeks because they have to order the parts and then fit it into their schedule. Servicing an oil sealed vacuum pump can be a messy business, their confidence in fixing it properly may not be high so it may be left to last.

- Send the vacuum pump back to the manufacturer or to a local repair company. You can have your own pump repaired or take what is often called a "pool pump" for a fast exchange. It is another pump that has already been repaired and tested and is in stock for fast turn around. (You may have your own spare pump.)

- Use a local vacuum pump service company to regularly check you vacuum pumps and change the oil on a regular schedule. They have the correct equipment to change the oil quickly and take away the old oil to save you the trouble of its safe disposal. If the pumps are maintained regularly, it may become apparent if one is not performing up to standards. Change out arrangements can be made in advance, and you stand less of a chance of an unexpected breakdown. A local company in eastern Pennsylvania is offering this service and their customers' say it works well.

Whichever option works best for you, plan ahead and keep records. If repairs are done on site order the spares kits, new oil and replacement filter elements before you need them. If your instrument is shut down for cleaning or refitting consider servicing the vacuum pumps while the other work is being carried out.

For further information on any of this discussion you are welcome to contact the author directly. Short seminars can be presented if your technicians want more vacuum technology knowledge. An excellent reference book for vacuum technology is "Modern Vacuum Practice, $3^{\text {rd }}$ edition." written and published by Nigel Harris in England, and stocked in the USA by Vacuum and Low Pressure Consulting.

Images courtesy of Edwards Vacuum

\section{Polishing Matters!}

\section{Flat Lapping \& Polishing}
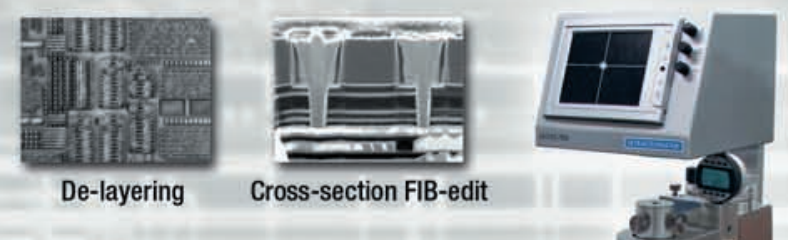

- Optical alignment makes flatness set-up

fast \& accurate

- Wide range of sample holders

- All polishing methods

\section{ULTRAPOL Advance}

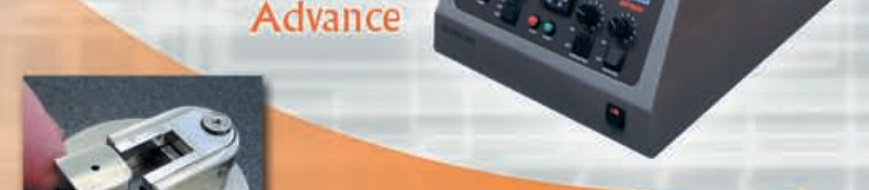

SEM \& FIB STUB HOLDERS Available for most microscopes

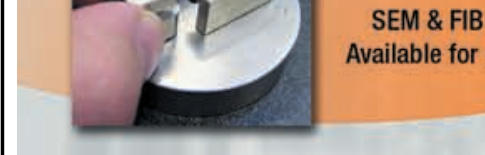

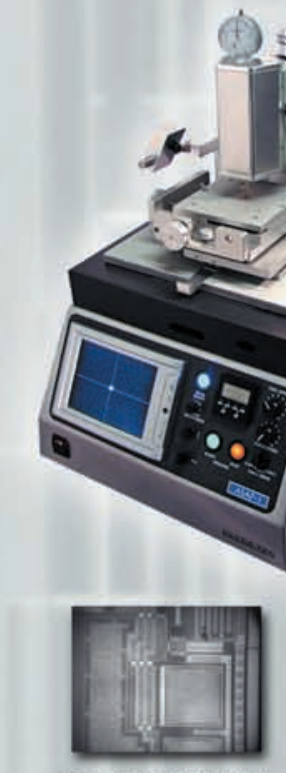

Backside Polishing

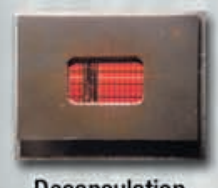

Decapsulation

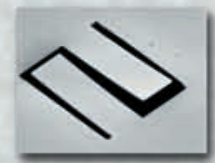

Thin Films (Courtesy of FIBICS, Inc.)
QURT TRA TTEC MANUIACTUासाNक. INC.

Toll Free (US) 1-877-542-0609

Tel: 1-714-542-0608 Fax: 1-714-542-0627

E-mail: info@ultratecusa.com www.ultratecusa.com 